\title{
Mesenchymal Motion Models in One Dimension
}

\author{
Zhian Wang ${ }^{1}$, Thomas Hillen ${ }^{2}$ and Michael $\mathrm{Li}^{2}$ \\ 1 Institute for Mathematics and its Applications, \\ University of Minnesota, Minneapolis, MN 55455, U.S.A \\ 2 Department of Mathematical and Statistical Sciences, \\ University of Alberta, Edmonton Alberta T6G 2G1, Canada
}

June 12, 2008

\begin{abstract}
Mesenchymal motion denotes a form of cell movement through tissue, which can be observed for certain cancer metastasis. In [11], a mathematical model for this form of movement was introduced. In the current paper we present a comprehensive analysis of the one dimensional mesenchymal motion model. We establish the global existence of classical solutions and rigorously carry out the parabolic limit of the model. We discuss the stationary solutions, prove the existence of travelling wave solutions, and we use numerical simulations to illustrate the results. Finally, we discuss the biological implications of the results.
\end{abstract}

Key words: Mesenchymal motion, pattern formation, global existence, macroscopic limits, traveling waves

\section{Introduction}

Mesenchymal motion is a form of cellular movement through tissues which are formed from fiber networks. An example is the invasion of tumor metastases through collagen networks [6]. Cells migrate in fiber networks and change their directions according to the orientational distribution of fibers. Moreover, cells actively remodel the matrix by excreting matrix degrading enzyme (e.g. protease) to generate sufficient space to migrate in.

Mesenchymal motion regarding the movement of amoeboid cells in a tissue matrix is reported in a review article by Friedl and Bröcker [6]. Mesoscopic and macroscopic models for mesenchymal motion were derived by Hillen [11] in a timely varying network tissue. The mesoscopic models are based on a transport equation for correlated random walk and consist of a transport equation for the cell movement coupled to an ordinary differential equation for the dynamics of tissue fibers. The macroscopic models have the form of a drift-diffusion equation where the mean drift velocity is given by the mean orientation of the tissue, and the diffusion 
tensor is given by the variance-covariance matrix of the tissue orientation. The analysis in [11] is divided into the case of undirected and directed tissues according to the distribution of fiber orientation. In undirected tissues, the fibers are symmetrical along their axis and both fiber directions are identical. For example, collagen fibers are undirected and they form the basis for many human and animal tissues. For directed tissues, the fibers are unsymmetrical and the two ends can be distinguished (such as microtubules and actin filaments). Branching collagen fiber networks can also be considered directional if the branching points are of significance for the movement of cells [11].

In this paper, the one dimensional mesenchymal motion model is fully analyzed. The global existence of solutions, macroscopic limits, traveling waves, and stationary solutions are investigated. The one dimensional model is very instructive and we can gain much insight into the mechanisms involved in the model. For example, we find the existence of traveling pulse solutions for the cell population and identify some mechanisms for cell aggregation. We restrict our attention to the model for directed tissue only and the analysis can be completely adopted to the study for undirected tissue from the mathematical point of view. The paper is organized as follows. In the rest of this section, we will present the one dimensional mesenchymal motion model derived in [11] and discuss the stationary solutions based on the telegraph process analysis. In section 2, we classify the one dimensional model as degenerated hyperbolic system and conclude that there is no shock solution. In section 3, the global existence of classical solutions are obtained along the characteristics using a fixed point argument and general regularity results for the semilnear hyperbolic system. In section 4 , we rigorously carry out the parabolic limit of the one dimensional mesenchymal transport model, where we show that solutions of the one dimensional model converge to solutions of a corresponding diffusion equation. In section 5 , we study the traveling wave solutions and find traveling pulse solutions for the cell population and travelling front waves for fiber orientations. In the final section 6 , we summarize and compare our results with the results obtained in [11]. Furthermore, we explain the findings in the context of the biological application of cell movement in tissues.

\subsection{Models for Mesenchymal Motion}

Here we keep notational consistence with [11], we let $S^{n-1}$ denote the unit sphere in $\mathbb{R}^{n}$ and $\theta$ the fiber orientation in $S^{n-1}$. Let $\Omega$ be a domain in $\mathbb{R}^{n}$. We denote the distribution of fiber orientations at time $t \geq 0$ and at location $x \in \Omega$ by $q(t, x, \theta)$, which naturally satisfies the normalization condition for all $t \geq 0, x \in \Omega$

$$
\int_{S^{n-1}} q(t, x, \theta) d \theta=1
$$

Let $V$ denote the set of all possible velocities of moving cells and $p(t, x, v)$ the population density of cells that have velocity $v$ at time $t$ at location $x . V$ is assumed to be radially symmetric and can be written as

$$
V=\left[s_{1}, s_{2}\right] \times S^{n-1}, \quad 0<s_{1} \leq s_{2}<\infty
$$


where $\left[s_{1}, s_{2}\right]$ is the range of possible speeds. We define $\hat{v}$ as the unit vector in direction of a vector $v \in V$. That is

$$
\hat{v}:=\frac{v}{\|v\|} .
$$

In addition we define a weight parameter $\omega$ such that

$$
\omega=\int_{V} q(t, x, \hat{v}) d v= \begin{cases}\frac{s_{2}^{n}-s_{1}^{n}}{n}, & \text { for } s_{1}<s_{2}, \\ s^{n-1}, & \text { for } s_{1}=s_{2}=s .\end{cases}
$$

Then $q / \omega$ is a probability density on $V$ and we will interpret the function $q(t, x, \theta) / \omega$ as probability density for the newly chosen movement direction of cells. Let a constant $\mu \geq 0$ denote the turning rate and a constant $\kappa \geq 0$ the cutting efficiency (rate of fiber degradation). Then the model for mesenchymal motion of cells for directed tissues consists of a transport equation for cell motion and an equation for the fiber distribution, which reads (see [11])

$$
\begin{aligned}
p_{t}(t, x, v)+v \cdot \nabla p(t, x, v) & =-\mu p(t, x, v)+\mu \frac{q(t, x, \hat{v})}{\omega} \rho(t, x), \\
q_{t}(t, x, \theta) & =\kappa(\Pi(t, x, \theta)-A(t, x)) \rho(t, x) q(t, x, \theta),
\end{aligned}
$$

where $\rho(t, x)$ denotes the macroscopic density of cells

$$
\rho(t, x)=\int_{V} p(t, x, v) d v
$$

and $\Pi(t, x, \theta)$ denote the mean projection of cell movement direction into a given fiber orientation $\theta$

$$
\Pi(t, x, \theta)=\frac{1}{\rho(t, x)} \int_{V} \theta \cdot \hat{v} p(t, x, v) d v,
$$

and $A(t, x, \theta)$ represents the mean value of these mean projections over all fiber directions

$$
A(t, x)=\int_{S^{n-1}} \Pi(t, x, \theta) q(t, x, \theta) d \theta
$$

The function $A(t, x)$ is a measure of the relative alignment of fibers and cells (see more details in [11]). The transport term $v \cdot \nabla p$ in (1.3) accounts for the cell migration in tissue with some certain velocity $v$. The right hand side of the first equation accounts for the change of cell movement in the field of fibers consisting of two processes. The first term on the right hand side describes turning of cells away from their velosity $v$ at a turning rate $\mu$. The second term indicates that cells choose a new direction $v$ which is given through the underlying fiber network $q(t, x, \bar{v})$ and they choose the speed uniformly from the interval $\left[s_{1}, s_{2}\right]$. The second euation of (1.3) describes the changes of the fibers in the tissue. When cells happen to migrate against the fiber direction, they cut those fibers at rate $\kappa$. At the same time, cells leave fibers intact that are parallel to their own movement direction. 
In [11], three scaling arguments, namely, moment closure, parabolic scaling and hydrodynamic scaling, are used to study the macroscopic limits of system (1.3). The resulting macroscopic models have the form of drift-diffusion equations with the mean drift velocity given by the mean orientation of the tissue and the diffusion tensor given by the variance-covariance matrix of the tissue orientations. Some examples and applications are discussed in [11]. The numerical schemes and pattern formation in two-dimension are elaborately investigated by Painter [22].

In case of chemotaxis, a system of a transport equation for the cell motion and a parabolic equation for the chemical signal was studied by Alt [1], Chalub et al. [3] and Hwang et al. $[15,16]$. However, their arguments are based on $L^{\infty}$ estimates of the turning kernel. In case of the mesenchymal motion model, the turning kernel is given by the fiber distribution $q(t, x, \theta)$ which is a delta distribution $q(\theta)=\delta_{b}(\theta)$ for a totally aligned tissue in some certain direction of $b \in \mathbb{R}^{n}(n \geq 2)$. As a result the assumption (A0) in paper [3] does not apply and hence their results cannot be applied directly to the case discussed here. The global existence analysis for the higher dimensional model is technically involved and will be explored in a separate paper [14], where we regard the solution as a measure.

The one-dimensional mesenchymal motion model corresponds to the case where the fiber are totaly aligned in the tissue. Below we present the one dimensional model derived in [11]. We fix speed to $|v|=s$, i.e., $v= \pm s$. In a one-dimensional domain, cells can only move to the right or the left. For notational convenience, we denote

$$
p^{+}(t, x)=p(t, x,+s), p^{-}(t, x)=p(t, x,-s),
$$

respectively. In one dimension, $S^{0}=\{+1,-1\}$ and hence $\theta= \pm 1$, and we denote

$$
q^{+}(t, x)=q(t, x,+1), q^{-}(t, x)=q(t, x,-1) .
$$

Then it follows from (1.1) that

$$
q^{+}(x, t)+q^{-}(x, t)=1 .
$$

The projection operator $\Pi(t, x, \theta)$ can be explicitly obtained in one dimension as

$$
\Pi(t, x, \theta)=\frac{1}{p^{+}+p^{-}}\left(\theta p^{+}-\theta p^{-}\right),
$$

which gives the following projection for right and left direction, respectively,

$$
\Pi^{ \pm}=\Pi(t, x, \pm 1)=\frac{p^{ \pm}-p^{\mp}}{p^{+}+p^{-}}
$$

Then the mean projection $A$ is obtained as

$$
A(t, x)=\Pi^{+} q^{+}+\Pi^{-} q^{-}=\frac{p^{+}-p^{-}}{p^{+}+p^{-}}\left(q^{+}-q^{-}\right) .
$$


Substituting all these results into the model (1.3), we obtain the one-dimensional mesenchymal transport model for the directed case (see [11])

$$
\begin{aligned}
p_{t}^{+}+s p_{x}^{+} & =-\mu p^{+}+\mu q^{+}\left(p^{+}+p^{-}\right), \\
p_{t}^{-}-s p_{x}^{-} & =-\mu p^{-}+\mu q^{-}\left(p^{+}+p^{-}\right), \\
q_{t}^{+} & =\kappa\left(p^{+}-p^{-}\right)\left(q^{-}-q^{+}+1\right) q^{+}, \\
q_{t}^{-} & =\kappa\left(p^{+}-p^{-}\right)\left(q^{-}-q^{+}-1\right) q^{-} .
\end{aligned}
$$

All above derivations are taken from paper [11] where more details are presented. It is worthwhile to point out that the model for undirected tissue can be regarded as a special case of (1.5) for $\kappa=0$ (see also [11]). In this paper, we focus on the model of directed tissue and most of reusults can be taken over to the case of undirected tissue. The significant difference, when it comes, will be emphasized.

Now we investigate the connections between the one dimensional mesenchymal motion model and the well known Goldstein-Kac model $[7,18]$ which describes the correlated random walk in one space dimension. We use condition (1.4) to substitute $q^{-}=1-q^{+}$into the first two equations of (1.5) and obtain

$$
\begin{aligned}
& p_{t}^{+}+s p_{x}^{+}=-\mu\left(1-q^{+}\right) p^{+}+\mu q^{+} p^{-}, \\
& p_{t}^{-}-s p_{x}^{-}=\mu\left(1-q^{+}\right) p^{+}-\mu q^{+} p^{-} .
\end{aligned}
$$

The model for the case of undirected tissue $(\kappa=0)$ possesses some very interesting phenomena. Undirected tisue fibers are symmetrical along their axis and both fiber directions are identical, which indicates that $q^{+}=q^{-}=\frac{1}{2}$. Then the model (1.6) becomes the Goldstein-Kac model $[7,18]$

$$
\begin{aligned}
& p_{t}^{+}+s p_{x}^{+}=\frac{\mu}{2}\left(p^{-}-p^{+}\right), \\
& p_{t}^{-}-s p_{x}^{-}=-\frac{\mu}{2}\left(p^{-}-p^{+}\right) .
\end{aligned}
$$

The parabolic scaling for the Goldstein-Kac model, which leads to a parabolic equation, has been discussed in [8] and references therein.

For directed tissues, we define $\lambda^{+}=\mu\left(1-q^{+}\right), \lambda^{-}=\mu q^{+}$, then (1.6) is converted into

$$
\begin{aligned}
& p_{t}^{+}+s p_{x}^{+}=-\lambda^{+} p^{+}+\lambda^{-} p^{-}, \\
& p_{t}^{-}-s p_{x}^{-}=\lambda^{+} p^{+}-\lambda^{-} p^{-},
\end{aligned}
$$

which is a modification of the Goldstein-Kac model. Extensions of the Goldstein-Kac model and local and global existence of the solution to the extended model has been extensively investigated in the literature $[12,13,17]$. The telegraph process of $(1.8)$ has been briefly discussed by Erban and Othmer [4] recently.The results obtained in [12, 13, 17] can be applied to system (1.8) if the turning rates $\lambda^{ \pm}(t, x)$ are given functions. The theory does, however, not apply to (1.5), since the turning rates are coupled with the $q^{ \pm}$equations. 
In the next subsection, we will discuss stationary solutions for (1.5) based on the telegraph process examined in [11].

We supply the system (1.5) with initial condition

$$
p^{ \pm}(0, x)=p_{I}^{ \pm}(x), q^{ \pm}(0, x)=q_{I}^{ \pm}(x), x \in \Omega .
$$

Due to the biological interest and condition (1.4), we make the following assumptions for the initial data and boundary conditions.

(ic) $p_{I}^{ \pm} \geq 0,0 \leq q_{I}^{+}, q_{I}^{-} \leq 1$ and $q_{I}^{+}+q_{I}^{-}=1$. For undirected tissues, we assume the initial data is symmetrical, i.e., $q_{I}^{+}=q_{I}^{-}=\frac{1}{2}$.

Here we consider two types of boundary conditions.

(bc1) $\Omega=\mathbb{R}$ and $p_{I}^{ \pm}(x), q_{I}^{ \pm}(x)$ have compact support in $\Omega$.

(bc2) $\Omega=[-l, l]$ and zero flux boundary condition, namely,

$$
p^{+}(t, \pm l)=p^{-}(t, \pm l)
$$

\subsection{Stationary Solutions}

In this section we discuss stationary solutions of the mesenchymal transport model (1.5) using a similar argument as used in [5]. We first present a second-order telegraph equation which is derived from system (1.5). To this end, we add and subtract the first two equations of (1.5) and obtain equations for the total population $p=p^{+}+p^{-}$and the population flux $j=s\left(p^{+}-p^{-}\right)$

$$
\begin{aligned}
p_{t}+j_{x} & =0, \\
j_{t}+s^{2} p_{x} & =-\mu j+\mu\left(q^{+}-q^{-}\right) s p .
\end{aligned}
$$

with initial conditions $p(0, x)=p_{I}(x)$ and $j(0, x)=j_{I}(x)$, where $p_{I}$ and $j_{I}$ are determined from the initial condition (1.9) of $p^{+}$and $p^{-}$. We differentiate the first equation of (1.10) with respect to $t$ and the second equation with respect to $x$. After that, we subtract the resulting equations and end up with a damped wave equation with drift term (see [11])

$$
p_{t t}+\mu p_{t}+\mu\left(s \xi_{q} p\right)_{x}=s^{2} p_{x x}
$$

where the drift velocity is given by the expectation of $q$ denoted by $\xi_{q}=q^{+}-q^{-}$. The equation (1.11) is a form of telegraph equation which describes electrical transmission in a telegraph cable when current leaks to the ground. A drift-diffusion equation can be approximated by taking the limit $\mu \rightarrow \infty, s \rightarrow \infty$ with diffusivity $D=s^{2} / \mu<\infty$ and drift velocity $s \xi_{q}<\infty$. The same drift-diffusion equation also can be obtained by multiscale methods (see [11]).

Suppose that equations (1.10) are defined in the interval $\Omega=[-l, l]$ and satisfies the boundary condition (bc2). In terms of cell population density, the zero flux boundary condition is equivalent to $p^{+}( \pm l)=p^{-}( \pm l)=\frac{1}{2} p( \pm l)$. We want to know under what conditions, if any, these equations have time-independent, nonconstant solutions for $p^{ \pm}$. The steady state condition $j_{x}=0$ of the first equation of (1.10) implies that $j$ is a constant and zero flux boundary 
condition $j( \pm l)=0$ furthermore gives that $j=0$. Consequently the second equation of (1.10) becomes

$$
p_{x}=\frac{\mu}{s}\left(q^{+}-q^{-}\right) p .
$$

This is a first order equation for $p$ whose solution can be easily found

$$
p(x)=p(-l) \exp \left(\frac{\mu}{s} \int_{-l}^{x}\left(q^{+}(y)-q^{-}(y)\right) d y\right) .
$$

The vanishing flux $j=0$ gives that $p^{+}=p^{-}$and hence

$$
p^{ \pm}(x)=\frac{p(-l)}{2} \exp \left(\frac{\mu}{s} \int_{-l}^{x}\left(q^{+}(y)-q^{-}(y)\right) d y\right) .
$$

Here the above integrals are bounded since $q^{+}$and $q^{-}$are bounded by 1 which will be proven later. From the above equations, one can see how the distribution of fiber orientations $q^{ \pm}$affect the distribution of cell population $p$ and $p^{ \pm}$. In particular, if $\mu \neq 0$ and $q^{+} \neq q^{-}$, then $p$ and $p^{ \pm}$ are nonconstants which correspond to the stationary solutions of the system (1.10).

Particulary in undirected tissues, $q^{+}=q^{-}=\frac{1}{2}$ due to symmetry, then $p$ and $p^{ \pm}$are constants and $p^{+}=p^{-}=\frac{p(-l)}{2}$, which means that there is no aggregation of cells.

If $q^{+}=1, q^{-}=0$, then

$$
p^{ \pm}(x)=\frac{p(-l)}{2} \exp \left(\frac{\mu}{s}(x+l)\right)
$$

The cells accumulate at the end $x=l$. This is not unexpected since all cells bias movement to the right and eventually accumulate at the right end due to zero-flux boundary condition.

Similarly, if $q^{+}=0, q^{-}=1$, then

$$
p^{ \pm}(x)=\frac{p(-l)}{2} \exp \left(-\frac{\mu}{s}(x+l)\right)
$$

and $p^{ \pm}$attains the maximum at $x=-l$.

Therefore here we identify a mechanism which can lead to aggregation, namely, $\mu \neq 0$ and the tissue are directed.

\section{Classification as Hyperbolic System}

We show that the system (1.5) is hyperbolic and we discuss shock solutions in this section. To this end, we rewrite (1.5) in a matrix form

$$
u_{t}+\Theta u_{x}=H(u)
$$

where $u, \Theta$ and $H(u)$ are defined as follows

$$
u=\left[\begin{array}{c}
p^{+} \\
p^{-} \\
q^{+} \\
q^{-}
\end{array}\right], \Theta=\left[\begin{array}{cccc}
s & 0 & 0 & 0 \\
0 & -s & 0 & 0 \\
0 & 0 & 0 & 0 \\
0 & 0 & 0 & 0
\end{array}\right], H(u)=\left[\begin{array}{c}
-\mu p^{+}+\mu q^{+}\left(p^{+}+p^{-}\right) \\
-\mu p^{-}+\mu q^{-}\left(p^{+}+p^{-}\right) \\
\kappa\left(p^{+}-p^{-}\right)\left(q^{-}-q^{+}+1\right) q^{+} \\
\kappa\left(p^{+}-p^{-}\right)\left(q^{-}-q^{+}-1\right) q^{-}
\end{array}\right]
$$


The drift term is linear and hence the system (2.1) cannot create shock solutions. The $4 \times 4$ matrix $\Theta$ has eigenvalues $\lambda_{1}=-s<0, \lambda_{2}=\lambda_{3}=0, \lambda_{4}=s$ satisfying $\lambda_{1}<\lambda_{2}=\lambda_{3}<\lambda_{4}$ provided that $s>0$. This implies the system (2.1) and hence (1.5) is hyperbolic but not strictly hyperbolic. The eigenvectors $r_{i}$ corresponding to eigenvalues $\lambda_{i}, i=1,2,3,4$ are

$$
r_{1}=\left[\begin{array}{l}
0 \\
1 \\
0 \\
0
\end{array}\right], r_{2}=\left[\begin{array}{l}
0 \\
0 \\
1 \\
0
\end{array}\right], r_{3}=\left[\begin{array}{l}
0 \\
0 \\
0 \\
1
\end{array}\right], r_{4}=\left[\begin{array}{l}
1 \\
0 \\
0 \\
0
\end{array}\right]
$$

It can be verified that $\nabla \lambda_{i}(u) \cdot r_{i}(u)=0$ for $i=1,2,3,4$, where $\nabla \lambda_{i}(u) \cdot r_{i}(u)$ means the directional derivative of the eigenvalues $\lambda_{i}$ in the direction of the eigenfunction $r_{i}$. Hence all characteristic field $\left(\lambda_{i}, r_{i}\right)$ are linearly degenerated $[2,20]$. Thus a shock which separates intersecting characteristics defining a discontinuity does not exist. However the solution might contain a contact discontinuity if data are discontinuous (see [2]).

The characteristic slopes are determined from the eigenvalues of the $4 \times 4$ matrix $\Theta$ in the

equation (2.1) by $\frac{d x}{d t}=\lambda_{i}$, which is never infinite, so the line $t=0$ is nowhere tangent to a characteristics. Therefore if initial data for $p^{+}, p^{-}, q^{+}, q^{-}$is given along the line $t=0$, the resulting Cauchy problem should be well-posed as verified in the next section.

\section{Global Existence}

In this section, we will prove the global existence of solutions to the system (1.5) subject to the initial condition (ic) and boundary condition (bc1). For bounded domain, the analysis for global existence will be a little bit more complicated than unbounded domain by taking into boundary conditions and is left open here.

The system (1.5) is a coupled system of two partial differential equations and two ordinary differential equations. To prove the global existence of solutions to the system (1.5), we first prove the nonnegativity of solutions.

Lemma 3.1. Let $p_{I}^{ \pm} \geq 0$ and $q_{I}^{ \pm} \geq 0$ with $q_{I}^{+}+q_{I}^{-}=1$. Assume that $p^{ \pm}, q^{ \pm} \in L^{\infty}\left(0, T ; L^{\infty}(\mathbb{R})\right)$ is a solution to system (1.5) for some $T>0$, then $p^{ \pm} \geq 0$ and $0 \leq q^{ \pm}(t, x) \leq 1$ with $q^{+}+q^{-}=1$.

Proof. We first show that $q^{+}+q^{-}=1$. Toward this end, we define two new quantities: $q=q^{+}+q^{-}, \xi=q^{+}-q^{-}$. Then we add and subtract the third and fourth equations of (1.5) to obtain equations for $q$ and $\xi$ as follows

$$
\begin{gathered}
q_{t}=-\kappa\left(p^{+}-p^{-}\right)(q-1) \xi, \\
\xi_{t}=\kappa\left(p^{+}-p^{-}\right)\left(q-\xi^{2}\right),
\end{gathered}
$$

which can be rewritten as a vector form

$$
Q_{t}=-\kappa\left(p^{+}-p^{-}\right) F(Q),
$$


where

$$
Q=\left(\begin{array}{c}
q \\
\xi
\end{array}\right), F(Q)=\left(\begin{array}{c}
(q-1) \xi \\
\xi^{2}-q
\end{array}\right)
$$

The initial data of the system (3.1) is given by

$$
q_{I}=q_{I}^{+}+q_{I}^{-}=1, \quad \xi_{I}=q_{I}^{+}-q_{I}^{-} .
$$

It is straightforward to verify that the vector field $F(Q) \in C^{1}\left(\mathbb{R}^{2}\right)$ and hence is locally Lipschitz continuous with respect to $Q$ for given $p^{ \pm} \in L^{\infty}\left(0, T ; L^{\infty}(\mathbb{R})\right)$. Then the Cauchy problem (3.1), (3.3) has a unique solution by the fundamental existence-uniqueness theorem. On the other hand, it is trivial to check that $q=1$ is a solution of the first equation (3.1) stasifying initial condition (3.3). Hence the system (3.1) and (3.3) has a unique solution $(q=1, \xi)$ where $\xi$ is determined by the equation

$$
\xi_{t}=\kappa\left(q^{+}-q^{-}\right)\left(1-\xi^{2}\right), \xi_{I}=q_{I}^{+}-q_{I}^{-} .
$$

It is worthwhile to point out that we provide an idea here to prove that $q=1$ and the existence (local) of $q$ and $\xi$ given that $p^{ \pm} \in L^{\infty}\left(0, T ; L^{\infty}(\mathbb{R})\right)$, which will be used later again without repeating this procedure.

We proceed to show that solutions $q^{ \pm}$preserve the positivity. Substituting $q^{-}=1-q^{+}$into the third equation of (1.5), we have

$$
q_{t}^{+}=2 \kappa\left(p^{+}-p^{-}\right)\left(1-q^{+}\right) q^{+}
$$

There are three cases to consider:

Case 1: $q_{I}^{+}=1$. Then we conclude that $q^{+}=1$ is a solution to equation (3.4) with initial condition $q_{I}^{+}=1$. Since the right hand side of equation (3.4) is locally Lipschitz continuous with respect to $q^{+}$, the solution of the equation (3.4) is unique. Hence $q^{+}(t, x)=1$ for all $t, x$.

Case 2: $q_{I}^{+}=0$. Using the similar argument as in Case 1 we can show that $q^{+}(t, x)=0$ is a unique solution to the equation (3.4).

Case 3: $0<q_{I}^{+}<1$. Then integrating the equation (3.4) with respect to $t$ from 0 to $t$, one has

$$
\frac{q^{+}}{1-q^{+}}=\frac{q_{I}^{+}}{1-q_{I}^{+}} \exp \left(\int_{0}^{t} 2 \kappa\left(p^{+}(\tau, \cdot)-p^{-}(\tau, \cdot)\right) d \tau\right) .
$$

Note that $0<q_{I}^{+}<1$. Then we have

$$
\frac{q^{+}}{1-q^{+}} \geq 0
$$

It follows immediately from the above equality that $0 \leq q^{+} \leq 1$. Combining Case 1, Case 2 and Case 3, we get that $0 \leq q^{+} \leq 1$ for $0 \leq q_{I}^{+} \leq 1$. Applying $q^{+}=1-q^{-}$into the fourth equation of (1.5) and using the same approach we can show that $0 \leq q^{-} \leq 1$. 
Finally we show the positivity of cell density $p^{ \pm}(t, x)$. We use a theory of invariant principle in paper [10] for the hyperbolic random walk system to achieve this goal. To this end, we write the first two equations of the system (1.5) in a matrix form

$$
\phi_{t}=G \phi+B \phi+\mathcal{F}(\phi)
$$

where

$$
\phi=\left(\begin{array}{c}
p^{+} \\
p^{-}
\end{array}\right), G=\left(\begin{array}{cc}
-s \frac{\partial}{\partial x} & 0 \\
0 & s \frac{\partial}{\partial x}
\end{array}\right), B=\left(\begin{array}{cc}
-\mu & \mu \\
\mu & -\mu
\end{array}\right)
$$

and

$$
\mathcal{F}(\phi)=\left(\begin{array}{c}
\mu q^{+}\left(p^{+}+p^{-}\right)-\mu p^{-} \\
\mu q^{-}\left(p^{+}+p^{-}\right)-\mu p^{+}
\end{array}\right)
$$

Let $\Lambda=[0, \infty) \subset \mathbb{R}$. Then $\Lambda$ is convex and for each $z \in \partial \Lambda, \Lambda$ has on outward normal vector. Moreover, define $\Sigma=\Lambda \times \Lambda$. Let $\phi \in \partial \Sigma$ and without loss of generality we assume that $\phi=(\vartheta, 0)$ with $\vartheta \geq 0$. Then for the outward normal vector $\eta(\phi)=(0,-1)$ of $\phi$, we have

$$
\eta(\phi) \cdot(B \phi+\mathcal{F}(\phi))=-\mu q^{-} \vartheta \leq 0,
$$

where we have used the positivity of $q^{-}$. Then by the theory in [10] (Theorem 2), the set $\Sigma$ is positively invariant for the system (3.5), which shows the positivity of $p^{ \pm}$. The proof is completed.

By the Lemma 3.1, we obtain the following theorem.

Theorem 3.1. The set $\left\{\left(p^{+}, p^{-}, q^{+}, q^{-}\right) \mid p^{ \pm} \geq 0, q^{ \pm} \geq 0, q^{+}+q^{-}=1\right\}$ is invariant to the system (1.5) provided that $p^{ \pm}, q^{ \pm} \in L^{\infty}\left(0, \infty ; L^{\infty}(\mathbb{R})\right)$.

Remark 3.1. For $p^{+}>p^{-}$, the term $p^{+}-p^{-}>0$ and $q^{+}$will increase while $q^{-}$decreases. Hence directionality is enhanced by the last two equations of (1.5).

Next, we are devoted to prove the global existence of solutions to system (1.5) subject to initial condition (ic). Due to Theorem 3.1, we can reformulate the system (1.5) as

$$
\begin{aligned}
p_{t}^{+}+s p_{x}^{+} & =-\mu p^{+}+\mu q^{+}\left(p^{+}+p^{-}\right), \\
p_{t}^{-}-s p_{x}^{-} & =-\mu p^{-}+\mu q^{-}\left(p^{+}+p^{-}\right), \\
\xi_{t} & =\kappa\left(p^{+}-p^{-}\right)\left(1-\xi^{2}\right),
\end{aligned}
$$

where $q^{+}$and $q^{-}$are given by

$$
q^{+}=\frac{1+\xi}{2}, q^{-}=\frac{1-\xi}{2} .
$$


It is worthwhile to note that here $\xi$ represents the expectation of fiber orientation in one dimension subject to the initial condition $\xi_{I}:=\xi(0)=q_{I}^{+}-q_{I}^{-}$. Furthermore from initial condition (ic), we have

$$
-1 \leq \xi_{I} \leq 1 .
$$

We seek the global solutions of the system (3.6) in the following space

$$
\mathbb{X}(0, T):=\left\{\left(p^{+}, p^{-}, \xi\right) \mid p^{ \pm}, \xi \in L^{\infty}\left(0, T ; L^{1} \cap L^{\infty}(\mathbb{R})\right)\right\}
$$

We first give the local existence of solutions for the system (3.6).

Lemma 3.2 (Local Existence). Let $p_{I}^{ \pm}, q_{I}^{ \pm}(x) \geq 0$ and $q_{I}^{+}+q_{I}^{-}=1$. Assume $p_{I}^{ \pm} \in L^{1} \cap L^{\infty}(\mathbb{R})$ and $\xi_{I} \in L^{1}(\mathbb{R})$. Then there exists a time $T_{0}>0$ such that the problem (3.6) with boundary condition (bc1) has a unique solution $\left(p^{+}, p^{-}, \xi\right) \in \mathbb{X}\left(0, T_{0}\right)$ satisfying $-1 \leq \xi \leq 1$.

Proof. For short we denote $\eta=\left(p^{+}, p^{-}, \xi\right)^{T}$. The norm of the vector $\eta$ is defined as

$$
\begin{aligned}
\|\eta\|_{L^{\infty}(\mathbb{R})} & =\max \left\{\left\|p^{+}\right\|_{L^{\infty}(\mathbb{R})},\left\|p^{-}\right\|_{L^{\infty}(\mathbb{R})},\|\xi\|_{L^{\infty}(\mathbb{R})}\right\}, \\
\|\eta\|_{L^{1}(\mathbb{R})} & =\max \left\{\left\|p^{+}\right\|_{L^{1}(\mathbb{R})},\left\|p^{-}\right\|_{L^{1}(\mathbb{R})},\|\xi\|_{L^{1}(\mathbb{R})}\right\},
\end{aligned}
$$

Moreover for the convenience of presentation we denote

$$
\begin{aligned}
& f_{1}\left(p^{+}, p^{-}, \xi\right)=-\mu p^{+}+\frac{\mu}{2}(1+\xi)\left(p^{+}+p^{-}\right), \\
& f_{2}\left(p^{+}, p^{-}, \xi\right)=-\mu p^{-}+\frac{\mu}{2}(1-\xi)\left(p^{+}+p^{-}\right), \\
& f_{3}\left(p^{+}, p^{-}, \xi\right)=\kappa\left(p^{+}-p^{-}\right)\left(1-\xi^{2}\right) .
\end{aligned}
$$

Clearly the function $f_{i}(i=1,2,3)$ is differentiable with repsect to its arguments and hence is locally Lipschitz continuous in any bounded subset of $L^{1} \cap L^{\infty}(\mathbb{R})$.

It is straightforward to show that system (3.6) is strictly hyperbolic with three distinct uniform bounded eigenvalues $\lambda_{1}, \lambda_{2}$ satisfying $-s=\lambda_{1}<\lambda_{3}=0<\lambda_{2}=s$. Then for each $i=1,2,3$ and each point $(t, x)$ in the $t-x$ plane, the characteristic equation of (3.6) defined by

$$
\frac{d \mathbf{x}_{i}}{d \tau}=\lambda_{i}, \mathbf{x}_{i}(t)=x
$$

has a unique solution defined for all $t>0$, describing the $i-t h$ characteristic through point $(t, x)$. We denote such a solution by $t \mapsto \mathbf{x}_{i}(\tau ; t, x)$, where $\mathbf{x}_{i}(\tau ; t, x)=x+\lambda_{i}(\tau-t)$ and in particular $\mathbf{x}_{3}(\tau ; t, x)=x$ due to $\lambda_{3}=0$. Following the argument in [2], we define

$$
\mathcal{D}=\{(t, x) \mid 0 \leq t<\ell / s,-\ell+s t \leq x \leq l-s t\} .
$$

Note that $\ell$ can be arbitrarily large since the domain is unbounded. Then for every $(t, x) \in \mathcal{D}$ and every $i \in\{1,2\}$, the characteristic curve $\left\{\left(t, x_{i}(\tau ; t, x)\right) \mid 0 \leq \tau \leq t\right\}$ is entirely contained inside $\mathcal{D}$ with $\mathbf{x}_{i}(0 ; t, x) \in[-\ell, \ell]$. Such a set $\mathcal{D}$ is called a domain of determinacy (see [2]). 
The system (3.6) has two independent characteristics. We integrate the first equation of (3.6) along the second characteristic curve $\mathbf{x}_{2}(\tau ; t, x)$, the second equation of (3.6) along the first characteristic $\mathbf{x}_{1}(\tau ; t, x)$ and the third equation along $\mathbf{x}_{3}(\tau ; t, x)=x,(3.6)$ can be rewritten as an ODE system

$$
\begin{aligned}
p_{\tau}^{+} & =-\mu p^{+}\left(\tau, \mathbf{x}_{2}(\tau)\right)+\mu q^{+}\left(\tau, \mathbf{x}_{2}(\tau)\right)\left(p^{+}\left(t, \mathbf{x}_{2}(\tau)+p^{-}\left(\tau, \mathbf{x}_{2}(\tau)\right)\right),\right. \\
p_{\tau}^{-} & =-\mu p^{+}\left(\tau, \mathbf{x}_{1}(\tau)\right)+\mu q^{-}\left(\tau, \mathbf{x}_{1}(\tau)\right)\left(p^{+}\left(\tau, \mathbf{x}_{1}(\tau)\right)+p^{-}\left(\tau, \mathbf{x}_{1}(\tau)\right)\right), \\
\xi_{\tau} & =\kappa\left(p^{+}(\tau, x)-p^{-}(\tau, x)\right)\left(1-\xi^{2}(\tau, x)\right)
\end{aligned}
$$

where $\mathbf{x}_{i}(\tau)=: \mathbf{x}_{i}(\tau ; t, x)$ for $i=1,2$ and $\mathbf{x}_{3}(\tau)=x$.

In a vector form, (3.8) can be reformulated as

$$
u_{\tau}=f(u), u \in \mathbb{R}^{3}
$$

where

$$
f(u)=\left(\begin{array}{c}
f_{1}\left(u\left(\tau, \mathbf{x}_{2}(\tau)\right)\right) \\
f_{2}\left(u\left(\tau, \mathbf{x}_{1}(\tau)\right)\right) \\
f_{3}(u(\tau, x))
\end{array}\right)
$$

Note that $\mathbf{x}_{i}(\tau) \in \mathbb{R}$ for $(i=1,2)$. Then $f(u)$ is locally Lipschitz continuous in any bounded subset of $L^{1} \cap L^{\infty}(\mathbb{R})$ and hence the local existence follows by the fundamental theorem of existence and uniqueness theorem(e.g., see [23]). Due to Theorem 3.1 and the definition of $\xi$, it has that $-1 \leq \xi \leq 1$. Then the proof is finished.

We proceed to derive a priori extimates in order to get global existence.

Lemma 3.3 (A priori extimates). Let the assumptions in Lemma 3.2 hold and $\left(p^{+}, p^{-}, \xi\right)$ be the solution obtained in Lemma 3.2. Then for any $0<t \leq T_{0}$, there exist constants $C>0$ and $\widetilde{C}>0$ such that

$$
\left\|p^{+}(t)\right\|_{L^{1} \cap L^{\infty}(\mathbb{R})}+\left\|p^{-}(t)\right\|_{L^{1} \cap L^{\infty}(\mathbb{R})}+\|\xi(t)\|_{L^{1} \cap L^{\infty}(\mathbb{R})} \leq C \exp (\widetilde{C} T),
$$

and $-1 \leq \xi \leq 1$, where $\|\cdot\|_{L^{1} \cap L^{\infty}(\mathbb{R})}=\|\cdot\|_{L^{1}(\mathbb{R})}+\|\cdot\|_{L^{\infty}(\mathbb{R})}$.

Proof. For each $(t, x) \in \mathcal{D}$ and $\mathbf{x}_{i}(0 ; t, x) \in[-\ell, \ell]$, we integrate the first two equations of (3.8) with respect to $\tau$ over $[0, t]$ and obtain that

$$
\begin{aligned}
p^{+}(t, x) & =p^{+}\left(\mathbf{x}_{2}(0)\right)+\int_{0}^{t} f_{1}\left(p^{+}\left(\tau, \mathbf{x}_{2}(\tau)\right), p^{-}\left(\tau, \mathbf{x}_{2}(\tau)\right), \xi\left(\tau, \mathbf{x}_{2}(\tau)\right)\right) d \tau \\
p^{-}(t, x) & =p^{-}\left(\mathbf{x}_{1}(0)\right)+\int_{0}^{t} f_{2}\left(p^{+}\left(\tau, \mathbf{x}_{1}(\tau)\right), p^{-}\left(\tau, \mathbf{x}_{1}(\tau)\right), \xi\left(\tau, \mathbf{x}_{1}(\tau)\right)\right) d \tau \\
\xi(t, \xi) & =\xi_{I}+\int_{0}^{t}\left(p^{+}(\tau, x)-p^{-}(\tau, x)\right)\left(1-\xi^{2}(\tau, x)\right) d \tau
\end{aligned}
$$


Note that we call $\left(p^{+}, p^{-}, \xi\right)$ a broad solution (see [2]) from the domain of determinacy $\mathcal{D}$ into $\mathbb{R}^{2}$ for Cauchy problem of $(3.8)$ if $\left(p^{+}, p^{-}, \xi\right)$ satisfies $(3.9)$, at almost every point $(t, x) \in \mathcal{D}$. Taking $L^{\infty}$-norm on both sides of of $(3.9)$, using the fact that $f_{i}$ is Lipschitz continuous, and tanking into account $f_{i}(0,0, \xi)=0$ for $i=1,2$, we infer that

$$
\begin{aligned}
& \left\|p^{+}(t)\right\|_{L^{\infty}(\mathbb{R})}+\left\|p^{-}(t)\right\|_{L^{\infty}(\mathbb{R})}+\|\xi(t)\|_{L^{\infty}(\mathbb{R})} \\
& \quad \leq C_{1}+C_{2} \int_{0}^{t}\left(\left\|p^{+}(\tau)\right\|_{L^{\infty}(\mathbb{R})}+\left\|p^{-}(\tau)\right\|_{L^{\infty}(\mathbb{R})}+\|\xi(\tau)\|_{L^{\infty}(\mathbb{R})}\right) d \tau .
\end{aligned}
$$

where $C_{1}$ is a constant such that $\left\|p_{I}^{+}\right\|_{L^{\infty}(\mathbb{R})}+\left\|p_{I}^{-}\right\|_{L^{\infty}(\mathbb{R})}+\left\|\xi_{I}\right\|_{L^{\infty}(\mathbb{R})} \leq C_{1}$ and $C_{2}$ depends on the Lipschitz contants of function $f_{i}(i=1,2,3)$ and the turing rate $\mu$.

The application of Gronwall's inequality to the above inequality gives

$$
\left\|p^{+}(t)\right\|_{L^{\infty}(\mathbb{R})}+\left\|p^{-}(t)\right\|_{L^{\infty}(\mathbb{R})}+\|\xi(t)\|_{L^{\infty}(\mathbb{R})} \leq C_{1} \exp \left(C_{2} t\right) .
$$

Similarly, one can deduce that there exist constants $C_{3}, C_{4}>0$ such that

$$
\left\|p^{+}(t)\right\|_{L^{1}(\mathbb{R})}+\left\|p^{-}(t)\right\|_{L^{1}(\mathbb{R})}+\|\xi(t)\|_{L^{1}(\mathbb{R})} \leq C_{3} \exp \left(C_{4} t\right) .
$$

The last two inequalities imply the first conclusion of the Lemma. The second conclusion follows from Theorem 3.1 and the definition of $\xi$.

By Lemma 3.2 and Lemma 3.3, the existence theorem of global solutions is obtained.

Theorem 3.2 (Global Existence). Let $q_{I}^{ \pm}(x) \geq 0$ and $q_{I}^{+}+q_{I}^{-}=1$. Assume $p_{I}^{ \pm}, \xi_{I} \in L^{1} \cap L^{\infty}(\mathbb{R})$. Then the problem (3.6) with boundary condition (bc1) has a unique global solution $\left(p^{+}, p^{-}, \xi\right) \in$ $\mathbb{X}(0, \infty)$ satisfying $-1 \leq \xi \leq 1$.

Proof. We suppose that the maximal time $T_{\max }$ of existence for the solution of (3.6) is finite, namely, $T_{\max }<\infty$. From Lemma 3.3 , we know that $-1 \leq \xi \leq 1$ for any $0 \leq t \leq T_{\max }$. Hence according to the well known alternative results (for example, see [21, 23]), one has that

$$
\lim _{t \rightarrow T_{\max }}\left\|p^{+}(t)\right\|_{L^{1} \cap L^{\infty}(\mathbb{R})}=\infty \quad \text { or } \quad \lim _{t \rightarrow T_{\max }}\left\|p^{-}(t)\right\|_{L^{1} \cap L^{\infty}(\mathbb{R})}=\infty .
$$

On the other hand, when $-1 \leq \xi \leq 1$, we have proven in Lemma 3.3 that for any $t \leq T_{\max }$, it holds that

$$
\left\|p^{+}(t)\right\|_{L^{1} \cap L^{\infty}(\mathbb{R})}+\left\|p^{-}(t)\right\|_{L^{1} \cap L^{\infty}(\mathbb{R})} \leq C \exp \left(\widetilde{C} T_{\max }\right), \quad 0 \leq t \leq T,
$$

which is contradictive to $(3.10)$ for $0<T_{\max }<\infty$. This contradiction, in turn confirms that $T_{\max }=\infty$ and hence the global solution follows.

Remark 3.2. Mathematically when cutting efficiency $\kappa=0$, the system (1.5) becomes the one dimensional mesenchymal motion model for undirected tissue (see [11]). Due to the assumption $q^{+}(t, x)=q^{-}(t, x)$ for undirected tissues, we obtain the following global theorem for the model associated with undirected tissue. 
Theorem 3.3. Let $\kappa=0$ and $q_{I}^{+}=q_{I}^{-}=1 / 2$. Assume $p_{I}^{ \pm} \in L^{1} \cap L^{\infty}(\mathbb{R})$. Then there exists a unique global solution to system (3.6) such that $\left(p^{+}, p^{-}, \xi\right) \in \mathbb{X}(0, \infty)$ satisfying $\xi=0$.

Since the functions on the right hand side of (1.5) are continuously differentiable with respect to $p^{+}, p^{-}, q^{+}$and $q^{-}$, by a theory for semilinear hyperbolic system in [2] (see Theorem 3.6 in $[2])$, the broad solution of Cauchy problem (1.5) obtained in Theorem 3.2 is indeed a classical solution provided that the initial data (1.9) is continuously differentiable, namely, we have the following results.

Theorem 3.4. Let the assumptions in Theorem 3.2 hold. In addition, we assume that the initial data in (1.9) are continuously differentiable. Then the broad solution $u: \mathcal{D} \rightarrow \mathbb{R}^{2}$ obtained in Theorem 3.2 provides a classical solution. Moreover, if initial data in (1.9) are nonnegative, the solution is nonnegative. Its partial derivatives $u_{t}, u_{x}$ are broad solutions of the following semilinear system, respectively,

$$
\begin{aligned}
\left(u_{t}\right)_{t} & =H_{u} u_{t}-\Theta \cdot\left(u_{t}\right)_{x}, \\
\left(u_{x}\right)_{t} & =H_{u} u_{x}-\Theta \cdot\left(u_{x}\right)_{x},
\end{aligned}
$$

where $u, H$ and $\Theta$ are defined as in section 2 and $H_{u}$ denotes the derivative of $H$ with respect to $u$.

Proof. The proof is similar to the argument in [2]. We omit the details.

\section{Macroscopic Limits}

For the given fiber distribution $q^{ \pm}(t, x)$, formal parabolic and hydrodynamic limits were derived in [11] for the mesenchymal motion models $(1.5)$ in $n(n \geq 1)$ dimensions. In this section we rigorously carry out the parabolic limits for system (1.5) under some suitable assumptions.

To derive a limiting diffusion model for (1.5), we use the parabolic scaling of space and time by $\bar{x}=\varepsilon x$ denoting a macroscopic space scale and $\bar{t}=\varepsilon^{2} t$ a long time scale. Substituting these transformations into (3.6) (or equivalently (1.5)) and dropping the bar for convenience, we obtain the following equations with initidal data

$$
\begin{aligned}
\varepsilon^{2} \partial_{t} p_{\varepsilon}^{+}+\varepsilon s \partial_{x} p_{\varepsilon}^{+} & =-\mu p_{\varepsilon}^{+}+\mu q_{\varepsilon}^{+}\left(p_{\varepsilon}^{+}+p_{\varepsilon}^{-}\right), \\
\varepsilon^{2} \partial_{t} p_{\varepsilon}^{-}-\varepsilon s \partial_{x} p_{\varepsilon}^{-} & =-\mu p_{\varepsilon}^{-}+\mu q_{\varepsilon}^{-}\left(p_{\varepsilon}^{+}+p_{\varepsilon}^{-}\right), \\
\varepsilon^{2} \partial_{t} \xi_{\varepsilon}^{+} & =\kappa\left(p_{\varepsilon}^{+}-p_{\varepsilon}^{-}\right)\left(1-\xi_{\varepsilon}^{2}\right), \\
p_{\varepsilon}^{ \pm}(0, \cdot) & =p_{I}^{ \pm}(\cdot), \xi_{\varepsilon}(0, \cdot)=q_{I}^{+}-q_{I}^{-},
\end{aligned}
$$

where

$$
q_{\varepsilon}^{+}=\frac{1+\xi_{\varepsilon}}{2}, q_{\varepsilon}^{-}=\frac{1-\xi_{\varepsilon}}{2}
$$


Note that the global existence of classical solutions to the above system for $\varepsilon=1$ has been established in section 4 and furthermore it holds that

$$
0 \leq q_{\varepsilon}^{+}, q_{\varepsilon}^{-} \leq 1,-1 \leq \xi_{\varepsilon} \leq 1
$$

Indeed the global existence of solutions to (4.1)-(4.4) and assertion (4.5) for each $\varepsilon>0$ can be obtained by directly adopting the argument for $\varepsilon=1$. Using (4.5) and denoting $J_{\varepsilon}=p_{\varepsilon}^{+}-p_{\varepsilon}^{-}$, we obtain by adding and subtracting (4.2) from (4.1)

$$
\begin{aligned}
\varepsilon^{2} \partial_{t} p_{\varepsilon}+\varepsilon s \partial_{x} J_{\varepsilon} & =0 \\
\varepsilon^{2} \partial_{t} J_{\varepsilon}+\varepsilon s \partial_{x} p_{\varepsilon} & =\mu \xi_{\varepsilon} p_{\varepsilon}-\mu J_{\varepsilon}
\end{aligned}
$$

with initial data $p_{\varepsilon}(0)=p_{I}=p_{I}^{+}+p_{I}^{-}, J_{\varepsilon}(0 t)=J_{I}=p_{I}^{+}-p_{I}^{-}$. The system (4.6), (4.7) is equivalent to the following second order damped hyperbolic equation (see also [11])

$$
\frac{\varepsilon^{4}}{\mu} \partial_{t}^{2} p_{\varepsilon}+\varepsilon^{2} \partial_{t} p_{\varepsilon}+\varepsilon \partial_{x}\left(s \xi_{\varepsilon} p_{\varepsilon}\right)=\varepsilon^{2} \frac{s^{2}}{\mu} \partial_{x}^{2} p_{\varepsilon}
$$

which indicates that the drift term is the dominating term for $\varepsilon$ small. As in [11], we assume that the expectation of fiber directions is small such that

$$
\xi_{q}(t, x)=\lim _{\varepsilon \rightarrow 0} \frac{1}{\varepsilon} \xi_{\varepsilon}\left(\frac{t}{\varepsilon^{2}}, \frac{x}{\varepsilon}\right)=\lim _{\varepsilon \rightarrow 0} \frac{1}{\varepsilon}\left[q^{+}\left(\frac{t}{\varepsilon^{2}}, \frac{x}{\varepsilon}\right)-q^{-}\left(\frac{t}{\varepsilon^{2}}, \frac{x}{\varepsilon}\right)\right]<\infty .
$$

Under the above assumption, we formally obtain a drift-diffusion model with diffusion coefficient $\frac{s^{2}}{\mu}$ and drift velocity $s \xi_{q}$ from (4.8) by sending $\varepsilon \rightarrow 0$ (see [11])

$$
\partial_{t} p+\partial_{x}\left(s \xi_{q} p\right)=\frac{s^{2}}{\mu} \partial_{x}^{2} p
$$

where $p$ is the limit of $p_{\varepsilon}$ as $\varepsilon \rightarrow 0$. The aim of this section is to show that the solution of equation (4.8) is convergent to the solution of equation (4.10) in the weak sense as $\varepsilon \rightarrow 0$. To proceed we give the definition of weak solutions we address here.

Definition 4.1. We say that a function $P \in L^{2}\left([0, T] ; H^{1}(\mathbb{R})\right)$ is a weak solution of (4.10) if $P(t, x)$ satisfies the following

(a) For any test function $\phi \in C_{0}^{\infty}([0, T) \times \mathbb{R})$, it holds that

$$
-\int_{0}^{T} \int_{\mathbb{R}} P \partial_{t} \phi d x d t-\int_{0}^{T} \int_{\mathbb{R}}\left(s \xi_{q} P\right) \partial_{x} \phi d x d t=\frac{s^{2}}{\mu} \int_{0}^{T} \int_{\mathbb{R}} P \partial_{x}^{2} \phi d x d t+\int_{\mathbb{R}} P_{I} \phi(0) d x .
$$

(b) $P(0)=p_{I}=p_{I}^{+}+p_{I}^{-}$. 
Next we establish the convergence properties of the solution $p_{\varepsilon}$ and $J_{\varepsilon}$ as $\varepsilon \rightarrow 0$. It suffices to derive uniform estimates for the solutions of system (4.6) and (4.7), which is given in the following Lemma.

Lemma 4.1. Let $p_{I}^{ \pm} \in H^{1}(\mathbb{R})$ and the assumption (4.9) hold. Assume further that there exists a constant $C_{1}>0$, independent of $\varepsilon$, such that

$$
\left|\xi_{\varepsilon}\right|,\left|\partial_{x} \xi_{\varepsilon}\right| \leq C_{1} \varepsilon
$$

Then there is a constant $C_{2}$, independent of $\varepsilon$, such that the solution $\left(p_{\varepsilon}, q_{\varepsilon}\right)$ of system (4.1)-(4.4) satisfies for any $0 \leq t \leq T$

$$
\begin{aligned}
& \left\|p_{\varepsilon}(t)\right\|_{H^{1}(\mathbb{R})}+\left\|J_{\varepsilon}(t)\right\|_{H^{1}(\mathbb{R})}+\left\|\varepsilon \partial_{t} p_{\varepsilon}\right\|_{L^{2}(\mathbb{R})} \\
& \leq C_{2}\left(C_{1}, \mu, T\right)\left(\left\|p_{I}\right\|_{H^{1}(\mathbb{R})}+\left\|J_{I}\right\|_{H^{1}(\mathbb{R})}\right)
\end{aligned}
$$

where the constant $C_{2}$ depends on $C_{1}, \mu$ and $T$.

Proof. We use the energy method to prove the Lemma. First note that $p_{\varepsilon}(0)=p_{I}=$ $p_{I}^{+}+p_{I}^{-} \in H^{1}(\mathbb{R})$ and $J_{\varepsilon}(0)=J_{I}=p_{I}^{+}-p_{I}^{-} \in H^{1}(\mathbb{R})$. Multiplying the equation (4.6) by $p_{\varepsilon}$ and the equation $(4.7)$ by $J_{\varepsilon}$, adding the resultant equations and integrating it over $[0, t) \times \mathbb{R}$, we end up with the following inequality

$$
\begin{aligned}
& \frac{1}{2} \int_{\mathbb{R}}\left(\left|p_{\varepsilon}\right|^{2}+\left|J_{\varepsilon}\right|^{2}\right) d x+\int_{0}^{t} \int_{\mathbb{R}} \mu \varepsilon^{-2}\left|J_{\varepsilon}\right|^{2} d x d \tau \\
& \quad=\frac{1}{2} \int_{\mathbb{R}}\left(\left|p_{I}\right|^{2}+\left|J_{I}\right|^{2}\right) d x+\int_{0}^{t} \int_{\mathbb{R}} \mu \varepsilon^{-2} \xi_{\varepsilon} p_{\varepsilon} J_{\varepsilon} d x d \tau \\
& \quad \leq \frac{1}{2} \int_{\mathbb{R}}\left(\left|p_{I}\right|^{2}+\left|J_{I}\right|^{2}\right) d x+\int_{0}^{t} \int_{\mathbb{R}} \mu C_{1}\left|\varepsilon^{-1} p_{\varepsilon} J_{\varepsilon}\right| d x d \tau,
\end{aligned}
$$

where we have used the assumption (4.11). Applying Young's inequality $\left|C_{1} \varepsilon^{-1} p_{\varepsilon} J_{\varepsilon}\right| \leq \frac{1}{2}\left(\varepsilon^{-2}\left|J_{\varepsilon}\right|^{2}+\right.$ $\left.C_{1}^{2}\left|p_{\varepsilon}\right|^{2}\right)$ in (4.13), we have

$$
\begin{aligned}
& \int_{\mathbb{R}}\left(\left|p_{\varepsilon}\right|^{2}+\left|J_{\varepsilon}\right|^{2}\right) d x+\int_{0}^{t} \int_{\mathbb{R}} \mu \varepsilon^{-2}\left|J_{\varepsilon}\right|^{2} d x d \tau \\
& \quad \leq \int_{\mathbb{R}}\left(\left|p_{I}\right|^{2}+\left|J_{I}\right|^{2}\right) d x+\mu C_{1}^{2} \int_{0}^{t} \int_{\mathbb{R}}\left|p_{\varepsilon}\right|^{2} d x d \tau .
\end{aligned}
$$

By Gronwall's inequality, we immediately get a $L^{2}$-estimate of $p_{\varepsilon}$ and $J_{\varepsilon}$ independent of $\varepsilon$ such that for $0 \leq t<T$

$$
\left\|p_{\varepsilon}\right\|_{L^{2}(\mathbb{R})}^{2}+\left\|J_{\varepsilon}\right\|_{L^{2}(\mathbb{R})}^{2} \leq\left(\left\|p_{I}\right\|_{L^{2}(\mathbb{R})}^{2}+\left\|J_{I}\right\|_{L^{2}(\mathbb{R})}^{2}\right) \exp \left(\mu C_{1}^{2} T\right)
$$

Next we go to the higher order estimates. To this end, we multiply the equation (4.6) by $-\partial_{x}^{2} p_{\varepsilon}$ and the equation (4.7) by $-\partial_{x}^{2} J_{\varepsilon}$. Then we end up with the following estimates using the same 
procedure as deriving (4.13)

$$
\begin{aligned}
& \frac{1}{2} \int_{\mathbb{R}}\left(\left|\partial_{x} p_{\varepsilon}\right|^{2}+\left|\partial_{x} J_{\varepsilon}\right|^{2}\right) d x+\int_{0}^{t} \int_{\mathbb{R}} \mu \varepsilon^{-2}\left|\partial_{x} J_{\varepsilon}\right|^{2} d x d \tau \\
& =\frac{1}{2} \int_{\mathbb{R}}\left(\left|\partial_{x} p_{I}\right|^{2}+\left|\partial_{x} J_{I}\right|^{2}\right) d x+\int_{0}^{t} \int_{\mathbb{R}}^{t} \mu \varepsilon^{-2} \partial_{x}\left(\xi_{\varepsilon} p_{\varepsilon}\right) \partial_{x} J_{\varepsilon} d x d \tau \\
& \leq \frac{1}{2} \int_{\mathbb{R}}^{t}\left(\left|\partial_{x} p_{I}\right|^{2}+\left|\partial_{x} J_{I}\right|^{2}\right) d x+\int_{0}^{t} \int_{\mathbb{R}} \mu C_{1} \varepsilon^{-1}\left(\left|p_{\varepsilon}\right|+\left|\partial_{x} p_{\varepsilon}\right|\right)\left|\partial_{x} J_{\varepsilon}\right| d x d \tau .
\end{aligned}
$$

Using Young inequality and the fact $(a+b)^{2} \leq 2\left(a^{2}+b^{2}\right)$ for $a, b \in \mathbb{R}$, we deduce that

$$
\begin{aligned}
& \int_{0}^{t} \int_{\mathbb{R}} \mu C_{1} \varepsilon^{-1}\left(\left|p_{\varepsilon}\right|+\partial_{x} p_{\varepsilon} \mid\right)\left|\partial_{x} J_{\varepsilon}\right| d x d \tau \\
& \leq \frac{1}{2} \int_{0}^{t} \int_{\mathbb{R}} \mu \varepsilon^{-2}\left|\partial_{x} J_{\varepsilon}\right|^{2} d x d \tau+\frac{C_{1}^{2}}{2} \int_{0}^{t} \int_{\mathbb{R}} \mu\left(\left|p_{\varepsilon}\right|+\left|\partial_{x} p_{\varepsilon}\right|\right)^{2} d x d \tau \\
& \leq \frac{1}{2} \int_{0}^{t} \int_{\mathbb{R}} \mu \varepsilon^{-2}\left|\partial_{x} J_{\varepsilon}\right|^{2} d x d \tau+C_{1}^{2} \int_{0}^{t} \int_{\mathbb{R}} \mu\left|\partial_{x} p_{\varepsilon}\right|^{2} d x d \tau+C\left(T, p_{I}, J_{I}\right),
\end{aligned}
$$

where (4.15) has been used and

$$
C\left(T, p_{I}, J_{I}\right)=\mu C_{1}^{2} T\left(\left\|p_{I}\right\|_{L^{2}(\mathbb{R})}^{2}+\left\|J_{I}\right\|_{L^{2}(\mathbb{R})}^{2}\right) \exp \left(\mu C_{1}^{2} T\right)
$$

Now substituting (4.17) into (4.13) and applying Gronwall's inequality into the resulting inequality, we infer that

$$
\begin{aligned}
& \left\|\partial_{x} p_{\varepsilon}\right\|_{L^{2}(\mathbb{R})}^{2}+\left\|\partial_{x} J_{\varepsilon}\right\|_{L^{2}(\mathbb{R})}^{2} \\
& \quad \leq C\left(T, p_{I}, J_{I}\right)\left(\left\|\partial_{x} p_{I}\right\|_{L^{2}(\mathbb{R})}^{2}+\left\|\partial_{x} J_{I}\right\|_{L^{2}(\mathbb{R})}^{2}\right) \exp \left(\mu C_{1}^{2} T\right) \\
& \quad \leq \mu C_{1}^{2} T\left(\left\|p_{I}\right\|_{H^{1}(\mathbb{R})}+\left\|J_{I}\right\|_{H^{1}(\mathbb{R})}\right)^{2} \exp \left(2 \mu C_{1}^{2} T\right)
\end{aligned}
$$

Furthermore by (4.6) we have

$$
\left\|\varepsilon \partial_{t} p_{\varepsilon}\right\|_{L^{2}(\mathbb{R})}=\left\|\partial_{x} J_{\varepsilon}\right\|_{L^{2}(\mathbb{R})} .
$$

Then the combination of (4.15), (4.18) and (4.19) gives (4.12) and completes the proof.

Theorem 4.1. Let the assumptions in Lemma 4.1 hold and $p_{\varepsilon}(0)=p_{I}=p_{I}^{+}+p_{I}^{-}$. Then as $\varepsilon \rightarrow 0$, the solutions $p_{\varepsilon}$ of the equation (4.8) converge to a limit function $p_{0}$, which is a weak solution of the equation (4.10) such that $p_{0}(t=0)=p_{I}$.

Proof. According to the energy estimates (4.12), we see that the solution sequence $p_{\varepsilon}$ is uniformly bounded in $L_{\text {loc }}^{2}\left([0, \infty) ; H^{2}(\mathbb{R})\right)$ and $\varepsilon \partial_{t} p_{\varepsilon}$ is uniformly bounded in $L_{\text {loc }}^{2}\left([0, \infty) ; L^{2}(\mathbb{R})\right)$ for every $\varepsilon>0$. 
As a consequence of the RELLICH-KONDRACHOV compactness theorem, there exist a subsequence of $p_{\varepsilon}$ and $\varepsilon \partial_{t} p_{\varepsilon}$, still denoted by $p_{\varepsilon}$ and $\varepsilon \partial_{t} p_{\varepsilon}$, and function $p_{0} \in L_{\mathrm{loc}}^{2}\left([0, \infty) ; H^{2}(\mathbb{R})\right)$ and $p_{1} \in L_{\text {loc }}^{2}\left([0, \infty) ; L^{2}(\mathbb{R})\right)$ such that

$$
\left\{\begin{array}{l}
p_{\varepsilon} \rightarrow p_{0} \quad \text { weakly in } L_{\text {loc }}^{2}\left([0, \infty) ; H^{1}(\mathbb{R})\right) \\
\varepsilon \partial_{t} p_{\varepsilon} \rightarrow p_{1} \quad \text { weakly in } L_{\text {loc }}^{2}\left([0, \infty) ; L^{2}(\mathbb{R})\right)
\end{array}\right.
$$

Next we show that the $p_{0}$ is a weak solution of the equation (4.10) subject to the given initial data. To this end we multiply the equation (4.8) by a test function $\phi \in C_{0}^{\infty}([0, T] \times \mathbb{R})$ with $\phi(T)=\partial_{t} \phi(T)=0$ and integrate the resultant equation to get

$$
\begin{aligned}
& \frac{\varepsilon^{2}}{\mu} \int_{0}^{T} \int_{\mathbb{R}} p \partial_{t}^{2} \phi d x d t+\frac{\varepsilon^{2}}{\mu} \int_{\mathbb{R}}\left[p_{\varepsilon}(T) \partial_{t} \phi(T)-\partial_{t} p_{\varepsilon}(0) \phi(0)\right] d x \\
& \quad-\frac{\varepsilon^{2}}{\mu} \int_{\mathbb{R}}\left[\partial_{t} p_{\varepsilon}(T) \phi(T)-p_{\varepsilon}(0) \partial_{t} \phi(0)\right] d x+\int_{0}^{T} \int_{\mathbb{R}} p_{\varepsilon} \partial_{t} \phi d x d t+\int_{\mathbb{R}} p_{\varepsilon}(T) \phi(T) d x \\
& \quad-\frac{1}{\varepsilon} \int_{0}^{T} \int_{\mathbb{R}}\left(s \xi_{\varepsilon} p_{\varepsilon}\right) \partial_{x} \phi d x d t=\int_{\mathbb{R}} p_{\varepsilon}(0) \phi(0) d x+\frac{s^{2}}{\mu} \int_{0}^{T} \int_{\mathbb{R}} p_{\varepsilon} \partial_{x}^{2} \phi d x d t
\end{aligned}
$$

Note that $p_{\varepsilon}(0)=p_{I}=p_{I}^{+}+p_{I}^{-} \in H^{1}(\mathbb{R})$. Hence $J \varepsilon(0)=J_{I}=p_{I}^{+}-p_{I}^{-} \in H^{1}(\mathbb{R})$ and $\varepsilon \partial_{t} p_{\varepsilon}(0)=\partial_{x} J_{\varepsilon}(0) \in L^{2}(\mathbb{R})$ from equation (4.6). Thus the second and third and fourth terms in (4.21) vanish as $\varepsilon \rightarrow 0$ by (4.20). Using assumption (4.9) and sending $\varepsilon \rightarrow 0$ in (4.21), we obtain from (4.20) that

$$
\begin{gathered}
\int_{0}^{T} \int_{\mathbb{R}} p_{0} \partial_{t} \phi d x d t-\int_{0}^{T} \int_{\mathbb{R}}\left(s \xi_{q} p_{0}\right) \partial_{x} \phi d x d t \\
=\int_{\mathbb{R}} p_{I} \phi(0) d x+\frac{s^{2}}{\mu} \int_{0}^{T} \int_{\mathbb{R}} p_{0} \partial_{x}^{2} \phi d x d t
\end{gathered}
$$

which shows that $p_{0}$ is a weak solution of the equation (4.10) satisfying the initial condition.

Remark 4.2. It is worthwhile to note that the assumption (4.11) and (4.9) is automatically satisfied for the case of undirected tissue where $\xi_{\varepsilon}=0$ (see also Remark 4.2). Then the limit equation for the case of undirected tissue is a pure diffusion equation without a drift term.

\section{$5 \quad$ Traveling Waves}

Since the system (1.5) models the invasion of cells through tissues, it is of interest to look for the traveling wave solutions for (1.5) and see what kinds of movement patterns are used by cells for invasion. To this end, we first use the invariant motion $q^{+}+q^{-}=1$ to rewrite system (1.5) as follows

$$
\begin{aligned}
p_{t}+j_{x} & =0 \\
j_{t}+s^{2} p_{x} & =-\mu j+\mu s\left(2 q^{+}-1\right) p, \\
q_{t}^{+} & =\frac{2 \kappa}{s} j\left(1-q^{+}\right) q^{+},
\end{aligned}
$$


where $p=p^{+}+p^{-}, j=s\left(p^{+}-p^{-}\right)$, as usual.

We introduce the wave variable

$$
z=x-c t,
$$

where $c \geq 0$ denotes the wave speed. Then we can define the wave profile by

$$
\begin{aligned}
p(z) & =p(t, x)=p(x-c t), \\
j(z) & =j(t, x)=j(x-c t), \\
q^{+}(z) & =q^{+}(t, x)=q^{+}(x-c t) .
\end{aligned}
$$

Substituting (5.2) into (5.1), we convert (5.1) into an ODE system as follows

$$
\begin{aligned}
-c p_{z}+j_{z} & =0, \\
-c j_{z}+s^{2} p_{z} & =-\mu j+\mu s\left(2 q^{+}-1\right) p, \\
-c q_{z}^{+} & =\frac{2 \kappa}{s} j\left(1-q^{+}\right) q^{+} .
\end{aligned}
$$

We prescribe the boundary conditions by

$$
\begin{aligned}
p(-\infty)=p(+\infty)= & 0, \\
j(-\infty)=j(+\infty)= & 0, \\
q^{+}(-\infty)=q_{l}^{+}, & q^{+}(+\infty)=q_{r}^{+},
\end{aligned}
$$

where $q_{l}^{+}$and $q_{r}^{+}$are constants and satisfy $0 \leq q_{l}^{+}, q_{r}^{+} \leq 1$ and $q_{l}^{-}>q_{r}^{+}$. That is, we look for the traveling pulse wave for $p$ and decreasing traveling front wave for $q^{+}$.

From equation (5.3) and the boundary conditions (5.4), we obtain an invariant of motion for $j$ and $p$

$$
j=c p \text {. }
$$

Then the system (5.3) is reduced to the following two dimensional system by the substitution of (5.5) into (5.3)

$$
\begin{aligned}
\left(c^{2}-s^{2}\right) p_{z} & =\mu p\left[c-s\left(2 q^{+}-1\right)\right] \\
q_{z}^{+} & =-\frac{2 \kappa}{s} p\left(1-q^{+}\right) q^{+}
\end{aligned}
$$

It is clear that (5.6) becomes a singular problem when $c=s$ and that this singular problem has no solution satisfying the boundary conditions (5.4). Indeed if $c=s$, then $q^{+}=1$ due to $\mu \neq 0$, which biologically means cells continuously move to the right without changing movement direction. Also, $q^{+}=1$ does not agree with the boundary conditions (5.4). Thus we assume $c \neq s$ hereafter. Consequently the system (5.6) can be rewritten as

$$
\begin{aligned}
p_{z} & =-\alpha p\left[c-s\left(2 q^{+}-1\right)\right] \\
q_{z}^{+} & =-\beta p\left(1-q^{+}\right) q^{+}
\end{aligned}
$$


where $\alpha=-\frac{\mu}{c^{2}-s^{2}}, \beta=\frac{2 \kappa}{s}>0$. Due to the biological interest, we only consider nonnegative solutions where $p \geq 0$ and $0 \leq q^{ \pm} \leq 1$. In fact, the nonnegativity of solutions to the system (5.7) with boundary conditions (5.4) can be analogously obtained by following the argument as used in section 3. Therefore we are only interested in those heteroclinic orbits that remain nonnegative.

\subsection{Phase Plane Analysis}

System (5.7) has a continuum of steady states $(0, \theta)$ with $0 \leq \theta \leq 1$. The Jacobian matrix linearized about the steady state $(0, \theta)$ is

$$
J_{s}=\left[\begin{array}{cc}
-\alpha(c-s(2 \theta-1)) & 0 \\
-\beta(1-\theta) \theta & 0
\end{array}\right] .
$$

The eigenvalues of $J_{s}$ are easily solved

$$
\lambda_{1}=-\alpha(c-s(2 \theta-1)), \lambda_{2}=0 .
$$

The corresponding eigenvectors are

$$
r_{1}=\left[\begin{array}{c}
\lambda_{1} \\
-\beta(1-\theta) \theta
\end{array}\right], r_{2}=\left[\begin{array}{l}
0 \\
1
\end{array}\right]
$$

When $c \neq s$, we have two cases to consider corresponding to the sign of eigenvalue $\lambda_{1}$.

Case 1. If $c>s>0$, then $\alpha<0$. It is straightforward to check that $\lambda_{1}>0$ which indicates every steady state $(0, \theta)$ with $0 \leq \theta \leq 1$ is unstable and consequently there is no nonnegative heteroclinic connection due to the lack of the stable manifold. We thus claim that $0 \leq c<s$ is a necessary condition for the existence of a traveling wave and $s$ is then a critical traveling speed. Thus we assume that $c<s$ here and hereafter.

Case 2. If $0 \leq c<s$, then $\alpha>0$. We first fix traveling speed $c$ and solve $c-s\left(2 \theta^{*}-1\right)=0$ to get $\theta^{*}=\frac{c+s}{2 s}$. Clearly we have that $0<\theta^{*}<1$. Furthermore the following arguments hold

$$
\begin{aligned}
& \theta<\theta^{*} \Rightarrow \lambda_{1}<0, \\
& \theta=\theta^{*} \Rightarrow \lambda_{1}=0, \\
& \theta>\theta^{*} \Rightarrow \lambda_{1}>0 .
\end{aligned}
$$

Next, we show that there exists a pair of equilibria which produce a heteroclinic connection for each fixed $c$ satisfying $0 \leq c<s$. From (5.8), we see that every steady state $(0, \theta)$ of the system (5.7) with $0 \leq \theta \leq 1$ has two manifolds one of which is a one dimensional center manifold corresponding to zero eigenvalue $\lambda_{2}$. Since each center manifold is invariant under the flow of the system (5.7) and the set $\left\{\left(p, q^{+}\right): p=0,0 \leq q^{+} \leq 1\right\}$ consists of steady states only and hence is invariant, the center manifold acts in the direction of the $q^{+}$axis where $0 \leq q^{+} \leq 1$. So the heteroclinic connection is only determined by the stable and unstable manifolds corresponding to positive and negative eigenvalue $\lambda_{1}$, respectively. The existence of a 
heteroclinic orbit connecting the unstable manifold of one fixed point with the stable manifold of another fixed point, corresponds to the existence of a traveling wave (heteroclinic orbit). We are going to rigorously prove the existence of such a heteroclinic connections below. Beyond this, we also shall prove the existence of a family of travelling waves since a continuum of steady state exists for the system (5.7). Before proceeding, we give a remark as follows.

Remark 5.1. The constants $q^{+}=0$ or $q^{+}=1$ are solutions of the second equation of (5.7) and furthermore it holds that

(a) If $q^{+}=0$, then $p \rightarrow+\infty$ as $z \rightarrow-\infty$.

(b) If $q^{+}=1$, then $p \rightarrow+\infty$ as $z \rightarrow+\infty$.

Therefore, neither the orbit $q^{+}=0$ nor $q^{+}=1$ can form a heteroclinic connection although $\left\{q^{+}=1\right\}$ is the unstable manifold of the equilibrium $(0,1)$ and $\left\{q^{+}=0\right\}$ is the stable manifold of the equilibrium $(0,0)$. So hereafter we assume that $0<q^{+}<1$ in order to study the existence of travelling waves.

\subsection{Existence of Travelling Waves}

To show that an unstable manifold can be connected by a stable manifold, we need to investigate the global structure of the original nonlinear system. Below we shall apply LaSalle's invariant principle (see $[9,19])$ to study the asymptotics of solutions of the system (5.7). The asymptotic behavior of solutions to the system (5.7) can be described in the following Lemma.

Lemma 5.1. Assume $0 \leq c<s$. Let $\left(p, q^{+}\right)$be a solution of (5.7) with initial conditions $p_{I}>0$ and $0<q_{I}^{+}<1$. Then the $\omega$-limit set is contained in the following set

$$
\mathbb{N}=\left\{\left(p, q^{+}\right) \mid p=0,0 \leq q^{+}<\theta^{*}\right\},
$$

and the $\alpha$-limit set is contained in the set

$$
\mathbb{G}=\left\{\left(p, q^{+}\right) \mid p=0, \theta^{*}<q^{+} \leq 1\right\},
$$

where $\theta^{*}=\frac{c+s}{2 s}$.

Proof. Define a function $V\left(p, q^{+}\right)$by $V\left(p, q^{+}\right)=q^{+}$. Then in the set $\left\{\left(p, q^{+}\right) \mid p \geq 0,0 \leq\right.$ $\left.q^{+} \leq 1\right\}, V\left(p(z), q^{+}(z)\right) \geq 0$ and $\frac{d V}{d z} \leq 0$ thanks to the second equation of (5.7). Given a number $L>0$, we now define a set

$$
\Omega_{L}=\left\{\left(p, q^{+}\right): V\left(p, q^{+}\right) \leq L, p>0,0<q^{+}<1\right\} .
$$

Since we restrict our attention to the case of $0<q^{+}<1$, we let $0<L<1$. Hence it holds that

$$
\Omega_{L}=\left\{\left(p, q^{+}\right): p>0,0<q^{+}<1\right\} .
$$

We now proceed to justify that the set $\Omega_{L}$ is bounded for given $0<L<1$. Toward this end, we divide the first equation of (5.7) by the second equation to obtain that

$$
\frac{d p}{d q^{+}}=-\frac{\alpha(c+s)}{\beta} \frac{1}{\left(1-q^{+}\right) q^{+}}+\frac{2 \alpha s}{\beta} \frac{1}{1-q^{+}} .
$$


Integrating (5.13) and recovering $\alpha$ and $\beta$ yield a first integral

$$
p\left(q^{+}\right)=\frac{\mu s}{2 \kappa}\left[\frac{\ln \left(1-q^{+}\right)}{c+s}-\frac{\ln q^{+}}{c-s}\right]+C,
$$

where $C$ is a constant of integration determined by the initial data of $q^{+}$given in (5.4).

Then for any $q^{+}=V\left(p, q^{+}\right)<L, p$ is bounded as a function of $q^{+}$from (5.14). As a result, the set $\Omega_{L}$ define above is bounded.

We now define another set

$$
\mathbb{N}_{1}=\left\{\left(p, q^{+}\right) \mid \frac{d V}{d z}=0,0<q^{+}<1\right\}
$$

From the second equation of (5.7), we know that

$$
\frac{d V}{d z}=0 \Longleftrightarrow p=0 \text { or } q^{+}=0 \text { or } q^{+}=1 .
$$

Therefore, $\mathbb{N}_{1}=\left\{\left(p, q^{+}\right) \mid p=0,0<q^{+}<1\right\}$ and is invariant since it is comprised of steady states only. With the help of LaSalle's invariant principle, the $\omega$-limits set of any trajectories of the system staring in the set $\Omega_{L}$ for $0<L<1$ is contained in the set $\mathbb{N}_{1}$. Indeed we can describe the asymptotic behavior of the solution more precisely. From (5.10), we know that $\lambda_{1}>0$ for all $\theta^{*}<\theta<1$. Then the equilibrium $(0, \theta)$ with $\theta^{*}<\theta<1$ is unstable. If we define $\mathbb{N}_{2}=\left\{\left(p, q^{+}\right) \mid p=0, \theta^{*}<q^{+}<1\right\}$, then all solutions of the system (5.7) converge to the set as $z \rightarrow+\infty$

$$
\mathbb{N}=\mathbb{N}_{1} \backslash \mathbb{N}_{2}=\left\{\left(p, q^{+}\right) \mid p=0,0 \leq q^{+} \leq 1\right\} .
$$

In a similar fashion, if we study the problem (5.7) backward on variable $z$, we can prove all solutions of (5.7) converge to the set $\mathbb{G}$ when $z \rightarrow-\infty$, which completes the proof.

The Lemma 5.1 shows that any trajectory of the system (5.7) starting in a neighborhood of an equilibrium $(0, \theta)$ with $\theta^{*}<\theta<1$ converges, as $z \rightarrow+\infty$, to another equilibrium $(0, \theta)$ with $0<\theta<\theta^{*}$, which gives a nonnegative heteroclinic orbit (traveling wave) connecting these two equilibria. This heteroclinic orbit can be explicitly given by a level curve equation (5.14). It is worthwhile to point out that the travelling speed $c$ can be 0 from our analysis, which corresponds to a standing wave.

Theorem 5.1. Let us consider the system (5.7). For each traveling speed $c$ with $0 \leq c<s$, and any $\theta^{*}<c_{1}<1$ there exists a bounded, nonnegative heteroclinic orbit connecting an equilibrium $\left(0, c_{1}\right)$ to the another equilibrium $\left(0, c_{2}\right)$, where $0<c_{2}<\theta^{*}$ with $\theta^{*}=\frac{c+s}{2 s}$. That is, there exists a traveling solution $\left(p, q^{+}\right)$of the system (5.7) connecting $\left(0, c_{1}\right)$ and $\left(0, c_{2}\right)$. Particularly, the system (5.7) admits a standing wave for $c=0$.

An example of traveling solution $\left(p, q^{+}\right)$for system (5.7) is numerically plotted in Figure 1. From the definition of $p$ and the relation (5.5), we can derive that

$$
p^{+}=\frac{s+c}{2 s} p, p^{-}=\frac{s-c}{2 s} p .
$$



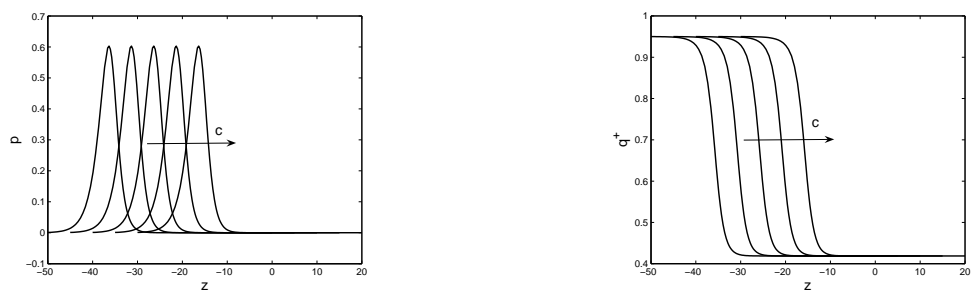

Figure 1: The traveling wave for the system (5.7), where $c=1, s=2, \mu=2, \kappa=1$. The waves travel from the left to the right and $c$ denotes the traveling speed and $z=0,5,10,15,20$.

In addition to the relation

$$
q^{-}=1-q^{+}, j=c p,
$$

we find the traveling waves for $p^{+}, p^{-}, q^{-}$and $j$ in terms of $p$ and $q^{+}$, as given above. The plot of the traveling structures of these quantities are given in Figure 2. A plot of all these quantities in a coordinate system is given in Figure 3 from which the transition properties between cell movement direction and fiber orinetation are clearly indicated.

From the equation of (5.1), we know that total mass of cells is conserved and so travelling pulse wave is expected as we found analytically and numerically above. The numerical simulation for $p$ in Figure 1 indicates that individual cells can move to the left or the right, but the whole cell group will move to right continuously. However, when the waves travel through, the fiber orientations are modified by cells and alignment to cell movement direction is enhanced, which is indicated by the numerical simulation for $q^{+}$in Figure 3.

\subsection{Family of Travelling Waves}

Note that for each left state $q_{l}^{+}$with $\theta^{*}<q_{l}^{+}<1$ we find a corresponding right state $\left(0, q_{r}^{+}\right)$ which is connected to $\left(0, q_{l}^{+}\right)$be a travelling wave with speed $c$. Here we give an explicit formula which related $q_{l}^{+}$and $q_{r}^{+}$.

Lemma 5.2. Given a speed $0 \leq c<s$. The left and right equilibria $\left(0, q_{l}^{+}\right)$and $\left(0, q_{r}^{+}\right)$are 

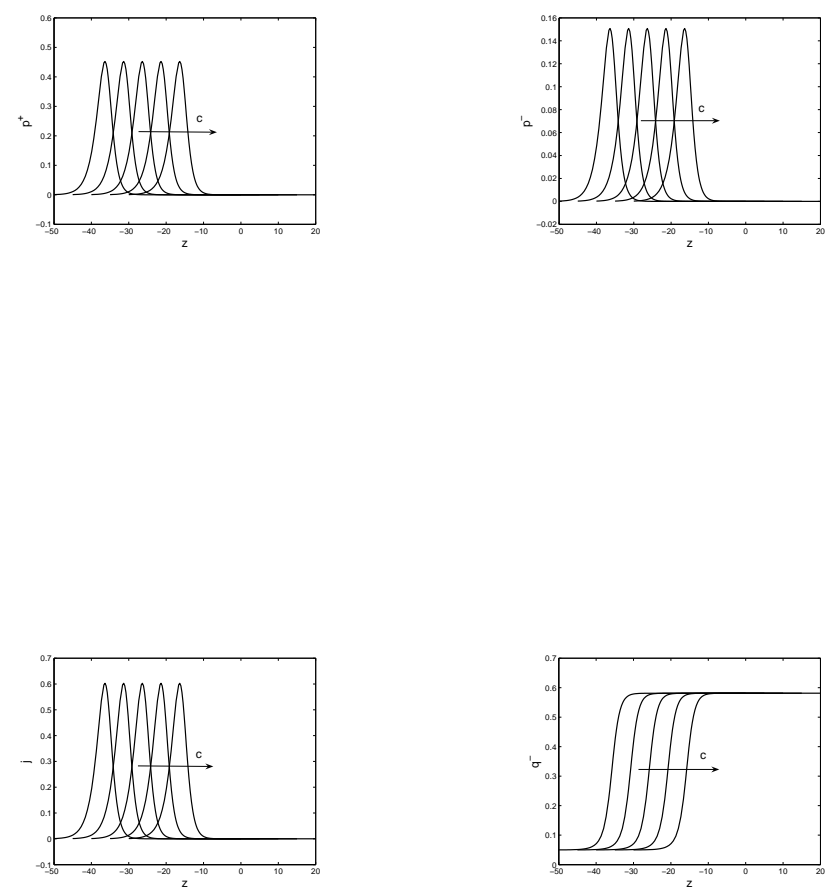

Figure 2: The numerical illustration of traveling waves for $p^{+}, p^{-}, j$ and $q^{-}$, where $c=1, s=$ $2, \mu=2, \kappa=1$. The waves shift from the left to the right and $c$ denotes the traveling speed and $z=0,5,10,15,20$. 


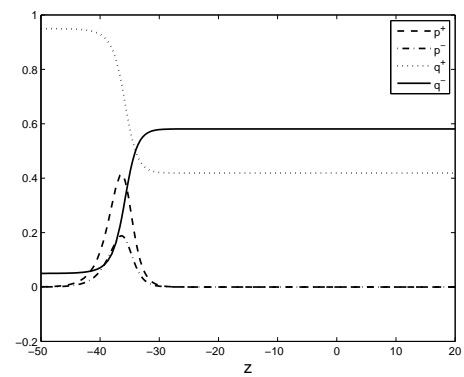

Figure 3: A plot of travelling solutions of system (1.5) in a coordinate system, where $c=1, s=$ $2, \mu=2, \kappa=1$ and $z=0,5,10,15,20$.

related as

$$
\left(\frac{1-q_{r}^{+}}{1-q_{l}^{+}}\right)^{s-c}=\left(\frac{q_{l}^{+}}{q_{r}^{+}}\right)^{s+c}, 0 \leq c<s,
$$

Proof. An explicit heteroclinic connection has been given by (5.14). By the Lemma 5.1, we infer that $p\left(q_{l}^{+}\right)=p\left(q_{r}^{+}\right)=0$. Applying this condition into (5.14), one has that

$$
\frac{\ln \left(1-q_{l}^{+}\right)}{c+s}-\frac{\ln q_{l}^{+}}{c-s}=\frac{\ln \left(1-q_{r}^{+}\right)}{c+s}-\frac{\ln q_{r}^{+}}{c-s} .
$$

Rearranging the above identity yields equation (5.17).

Hence we observe a family of heteroclinic orbits as shown in Figure 4.

From (5.14) we see that $p$ is bounded as a function of $q^{+}$if $0<q^{+}<1$. It would be also of interest to find the upper bound for each orbit and see how the upper bound varies with respect to the right/left states of $q^{+}$. Indeed, by (5.13), we get a unique critical point $q^{+}=\theta^{*}$ such that $\left.\frac{d p}{d q^{+}}\right|_{q^{+}=\theta^{*}}=0$. The second derivative of $p$ with respect to $q^{+}$is

$$
\frac{d^{2} p}{d q^{+^{2}}}=-\frac{\mu s}{2 \kappa}\left[\frac{1}{(c+s)\left(1-q^{+}\right)^{2}}+\frac{1}{(s-c) q^{+^{2}}}\right]
$$




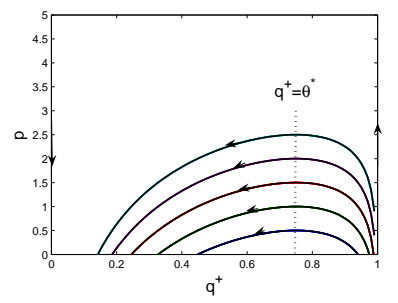

Figure 4: The illustration of a family of heteroclinic orbits for the system (5.7), where $c=1, s=$ $2, \mu=2, \kappa=1$ and $\theta^{*}=0.75$. The arrow denotes the orientation of trajectories of the system $(5.7)$.

Noting that $0 \leq c<s$. It is easy to verify that $\frac{d^{2} p}{d q^{+^{2}}}<0$ at $q^{+}=\theta^{*}$. Moreover we know that $p\left(q_{l}^{+}\right)=p\left(q_{r}^{+}\right)=0$. Hence $p$ attains the maximal value at $q^{+}=\theta^{*}$ given by

$$
p_{\max }=\frac{\mu s}{2 \kappa}\left[\frac{\ln \left(1-\theta^{*}\right)}{c+s}-\frac{\ln \theta^{*}}{c-s}\right]+\sigma
$$

where

$$
\sigma=-\frac{\mu s}{2 \kappa}\left[\frac{\ln \left(1-q_{l}^{+}\right)}{c+s}-\frac{\ln q_{l}^{+}}{c-s}\right], \theta^{*}=\frac{c+s}{2 s} .
$$

Remark 5.2. From the above equation, we know that the upper bound $p_{\max }$ of $p$ depends on the left states $q_{l}^{+}$of $q$. Also, we can easily verify that upper bound $p_{\max }$ increases with respect to $q_{l}^{+}>\theta^{*}$ (see Figure 4).

Remark 5.3. The results obtained above for traveling waves are only valid for the case of directed tissues. For undirected tissues, travelling waves with $c<s$ do not exist. Indeed, in the undirected case, we know that $q^{+}=q^{-}=\frac{1}{2}$ and the system (5.7) is reduced to a scalar equation

$$
p_{z}=\frac{\mu^{2}}{c^{2}-s^{2}} c p
$$


Clearly, the equation (5.21) has no solution satisfying boundary conditions (5.4).

\section{Conclusions}

In this paper, we analyze the one dimensional mesenchymal motion model proposed by Hillen [11]. We establish the global existence of classical solutions for both case of directed and undirected tissue. Particularly, we show that the model of undirected tissues $(\kappa=0)$ has a constant solution for fiber orientation distribution such that $q(t, x,+s)=q(t, x,-s)=\frac{1}{2}$, which means cells have no preference in choosing a particular movement direction and they have equal probability to move to the right or left. We discuss the existence of inhomogeneous steady sates for the case of directed tissue. We rigorously show the convergence of macroscopic limits of the model, i.e., the solutions of the mesoscopic model convergence to the corresponding macroscopic continuum model. Moreover, we study the traveling wave solutions and establish the existence of traveling pulse in total cell population $p(t, x)$ and traveling front waves in fiber orientation distribution $q^{ \pm}(t, x)$. The standing wave $(c=0)$ is admitted in our analysis. This is not unexpected since cells can move in two directions (left and right) and two traveling waves with opposite direction can eliminate each other to result in a standing wave. All our results are fairly consistent with the biological relevance discussed in paper [11].

The one-dimensional model appears artificial if compared to the real three-dimensional process of cell movement in fiber tissues. The benefit of studying the one-dimensional model in detail is twofold. First of all, this model and it's properties give good intuition into mechanisms that might be important in the higher dimensional case. For example the existence of non-homogeneous steady states will also be expected for higher dimensional models. Also, the model with directed fibers seems to have a richer behavior and admits traveling pulses, whereas the undirected model does not have traveling pulses. The same might be true in higher dimensions. Secondly, the model considered here describes cell movement in highly aligned tissue. In fact, many tissues show a predominant orientation, for example the white matter tracks inside brain tissue. The model studied here can be used to describe spread and propagation along those aligned tissues. In that case, the traveling pulse waves from section 5 correspond to an application of a "comb" to tissue that is aligned positively or negatively in a common direction. If a brush is applied upstream, say, the fibers will be flipped and a higher alignment to the right results (see simulations).

For the application of these models to cancer invasion through collagen tissue, the undirected formalism is important. The result of no traveling pulses for that case does not preclude invasions. It only precludes invasion in a self similar fashion. It is still possible that cells invade new areas, in particular if non-linear proliferation terms are added. Traveling waves under incorporation of cell proliferation is an interesting open question that comes out of the research done here.

Mathematically, the higher dimensional mesenchymal motion models show significant differences to the one dimensional case. In one dimension, fiber orientation $q(t, x, \theta)$ only has two direction and hence is bounded due to the normalization condition (5.1). However, in higher di- 
mension, fibers have infinite many distributional directions and highly aligned tissue corresponds to $q(t, x, \theta)$ being a Dirac delta function along that direction. Hence the function spaces have to be chosen to include non-integrable distributions, and standard $L^{2}$ or $L^{\infty}$ methods do not apply. In a forthcoming paper [14], we will study the existence of solutions for the high dimensional mesenchymal motion models in a Banach space of measurable functions using semigroup theory. If the existence theory stands, we can look into the interesting network formation dynamics, which were found numerically in Painter [22].

\section{Acknowledgment}

We thank Dr. D. Wrzosek at the Warsaw University for the very stimulating discussion. The first author is supported by the IMA postdoctor fellowship at the University of Minnesota and the second and third author are supported by the Natural Science and Engineering Council of Canada (NSERC).

\section{References}

[1] W. Alt. Biased random walk model for chemotaxis and related diffusion approximation. J. Math. Biol., 9:147-177, 1980.

[2] A. Bressan. Hyperbolic System of Conservation Laws: the One-dimensional Cauchy Problem. Oxford University Press, New York, 2001.

[3] F. Chalub, P.A. Markowich, B. Perthame, and C. Schmeiser. Kinetic models for chemotaxis and their drift-diffusion limits. Monatsh. Math., 142:123-141, 2004.

[4] R. Erban and H. Othmer. From signal transduction to spatial pattern formation in E. coli: a paradigm for multiscale modeling in biology. Multiscale Model. Simul., 3(3):362-394, 2005 .

[5] R. Erban and H. Othmer. Taxis equations for amoeboid cells. J. Math. Biol., 54:847-885, 2007.

[6] P. Friedl and E.B. Bröcker. The biology of cell locomotion within three dimensional extracellular matrix. Cell. Mol. Life Sci., 57:41-64, 2000.

[7] S. Goldstein. On diffusion by discontinuous movements and the telegraph equation. Quart. J. Mech. Appl. Math., 4:129-156, 1951.

[8] K.P. Hadeler. Reaction transport systems in biological modelling. In V. Capasso and O. Diekmann, editors, Mathematics Inspired by Biology, Lect. Notes Math. 1714, pages 95-150, Heidelberg, 1999. Springer Verlag.

[9] J.P. Hespanha. Uniform stability of switched linear systems: Extensions of lasalle's invariant principle. IEEE transactions on Automatic Control., 49(4):470-482, 2004. 
[10] T. Hillen. Invariant principle for hyperbolic random walk systems. J. Math. Anal. Appl, 210:360-374, 1997.

[11] T. Hillen. $M^{5}$ mesoscopic and macroscopic models for mesenchymal motion. J. Math. Biol., $53: 585-616,2006$.

[12] T. Hillen, C. Rohde, and F. Lutscher. Existence of weak solutions for a hyperbolic model for chemosensitive movement. J. Math. Anal. Appl., 260:173-199, 2001.

[13] T. Hillen and A. Stevens. Hyperbolic models for chemotaxis in $1-$ D. Nonlinear Analysis: Real World Applications, 1(1):409-433, 2001.

[14] P. Hinow, Z. Wang, and T. Hillen. Global existence for mesenchymal transport models in higher dimensions. In preparation, 2007.

[15] H.J. Hwang, K. Kang, and A. Stevens. Drift-diffusion limits of kinetic models for chemotaxis: a generalization. Discrete Contin. Dyn. Syst. Ser. B, 5:319-334, 2005.

[16] H.J. Hwang, K. Kang, and A. Stevens. Global solutions of nonlinear transport equations for chemosensitive movement. SIAM J. Math. Anal., 36:1177-1199, 2005.

[17] H.J. Hwang, K. Kang, and A. Stevens. Global existence of classical solutions for a hyperbolic chemotaxis model and its parabolic limit. Indiana Univ. Math. J., 55:289-316, 2006.

[18] M. Kac. A stochastic model related to the telegrapher's equation. Rocky Mountain J. Math., 4:497-509, 1956.

[19] J.P. LaSalle. The Stability of Dynamical Systems, ser. Regional conference series in applied mathematics. Society for Industrial and Applied Mathematics, 1976.

[20] P.G. LeFloch. Hyperbolic System of Conservation Laws: the Theory of Classical and Nonclassical Shock Waves, Lectures in mathematics. ETH Zürich, Birkhäuser Verlag, BaselBoston-Berlin, 2002.

[21] H. Matano. Convergence of solutions of one-dimensional semilinear parabolic equations. $J$. Math. Kyoto. Univ, 18:221-227, 1978.

[22] K. Painter. Modelling cell migration strategies in the extracelluar matrix. Submitted, 2007.

[23] J.C. Robinson. Infinite-Dimesnsional Dynamical Systems: an introduction to dissipative parabolic PDEs and the theory of global attractors. Cambridge University Press, Cambridge, 2001. 